\title{
An Environment to Support Micro-Incremental Class Development ${ }^{\dagger}$
}

\author{
Allen Parrish \\ David Cordes \\ Dennis Brown
}

The University of Alabama

Department of Computer Science

Box 870290

Tuscaloosa, AL 35487

July 1995

\begin{abstract}
Incremental development and testing is widely cited as one advantage of the object-oriented paradigm. To date, most of the work in this area emphasizes incremental development at the "macro" level, i.e., at the application or class hierarchy levels. We believe that incremental development should also be exploited at the individual class level. In particular, classes may contain a variety of methods that place objects of the class into relatively complex states. By organizing and developing an individual class in an incremental fashion, one can (a) develop and test "partial classes" and (b) generate simple states for test objects prior to generating more complex ones. This process realizes two benefits: it simplifies debugging by reducing the size of the search space when tracking down defects, and it makes regression testing more efficient.

This paper reports on a development environment designed to support "micro-incremental" class development. This environment integrates several different components and techniques. We discuss each component of the environment individually, and then illustrate the use of the environment in a case study.
\end{abstract}

\footnotetext{
${ }^{\dagger}$ Research supported in part by the National Science Foundation under grant CDA-9322010.
} 


\section{An Environment to Support Micro-Incremental Class Development}

\section{Introduction}

Incremental development and testing is widely cited as one of the many advantages in the objectoriented paradigm. However, most previous work emphasizes incremental development at the "macro" level, i.e., at the application or class hierarchy levels [4]. Because individual classes are themselves often nontrivial, we believe that it is useful to define "micro-incremental" techniques to be used when developing and testing individual classes. Such techniques allow the developer to build and test subsets of class operations, rather than waiting until after the class is written to begin testing (as is the case with most existing class testing methods $[1,2,5,6,11]$ ).

In this paper, we present an environment to support micro-incremental class development. Our environment utilizes formal specifications, requiring that the class under test be algebraically specified [3, 7]. The environment uses a formal specification language called LOBAS [1]. Our environment synthesizes three major components:

- A class generator,

- A development ordering generator, and

- A test oracle generator.

The class generator produces a partial $\mathrm{C}++$ class from a LOBAS specification. While the entire implementation is not produced, a structural template for the class is generated. The development ordering generator produces a recommended ordering of the class's methods, presenting the order in which the methods should be developed in order to maximize the amount of testing which can be performed during development. Finally, the test oracle generator produces a driver program which executes a series of test cases on the class, providing results in terms of whether a given test case "passes" or "fails."

The remainder of this paper is organized as follows. Section 2 provides an overview of the basic framework used in class testing with algebraic specifications. Section 3 introduces the concept of micro-incremental development and presents some micro-incremental development techniques. Section 4 then introduces our environment and describes how the environment can be used to construct a debugged implementation for a formally specified class.

\section{A Basic Framework}

\subsection{Object-Oriented Programming and Algebraic Specifications}

Our model of object-oriented programming is similar to the model used in the object-oriented test-

ing discussion in [1]. We use the term class to refer to the implementation of an abstract data type within an object-oriented language. An object is an instance of a class. A class implementation 
is defined in two parts: an interface consisting of a list of operations that can be performed on instances of the class, and a body consisting of the implementation of the operations. The implementation of an operation is sometimes called a method, and invoking an operation with respect to a given object is sometimes referred to as "sending a message" to the object, which responds to the message by executing its method. Additionally, every object has a state, which may be characterized by its history of method invocations.

Numerous class testing techniques have been developed for classes that have been formally specified using algebraic specification techniques. The ordered list class in Table 1 is specified using a language called LOBAS [1].

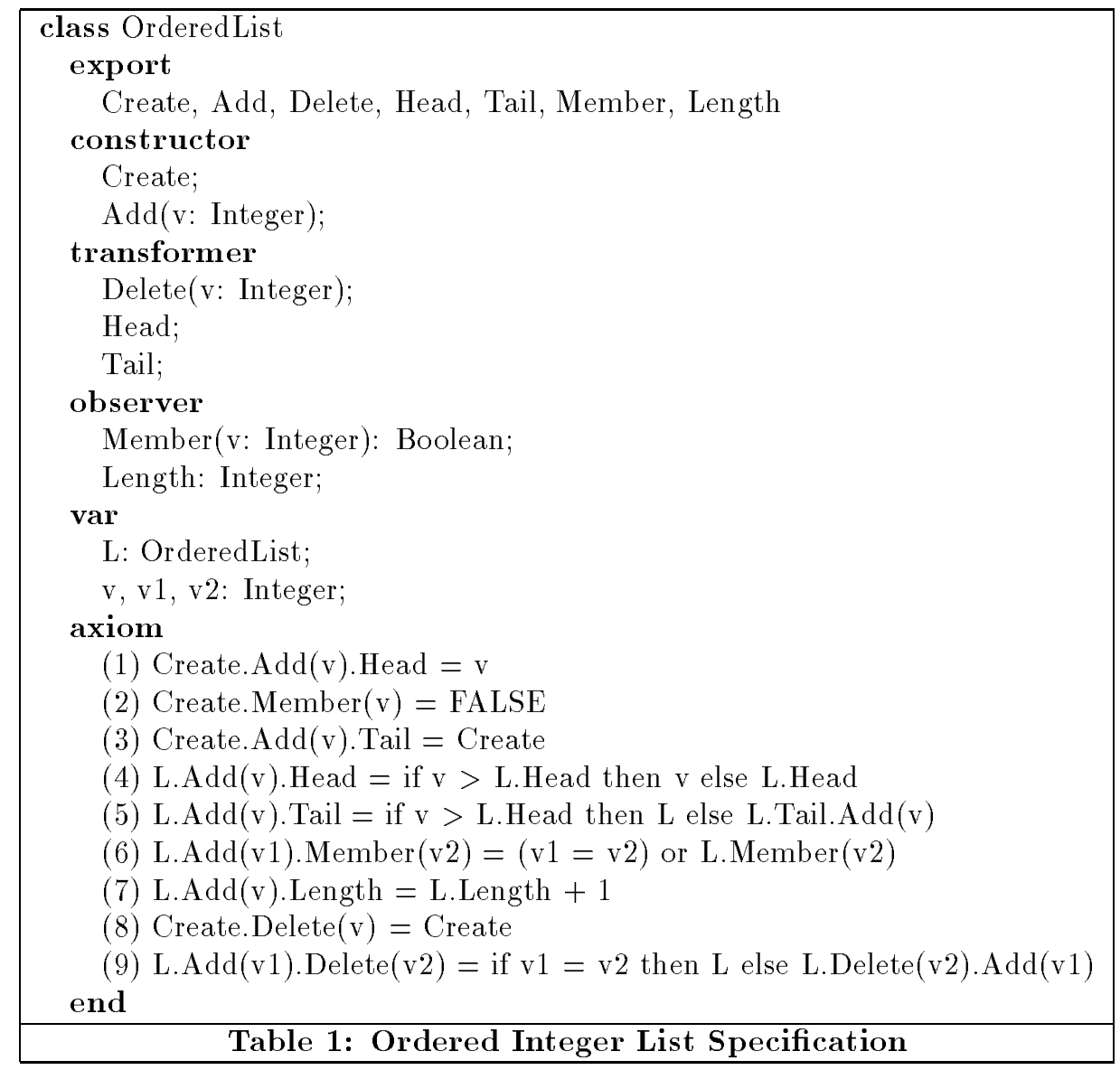

The specification requires that all methods be categorized as either a constructor, transformer, or observer. Observer methods return a type other than that of the class itself; the purpose of an observer method is to query the contents of an object (e.g., IsEmpty, Length, Top). Constructor and transformer methods both return objects of the class itself. The distinction between constructors and transformers is somewhat subtle and is explained below.

The axioms define the semantics of the class. Sequences of methods separated by dots are read left to right. For example, the sequence "Create.Add(10). Tail" means that Create is first executed; Add(10) is then executed on the result returned by Create, and Tail is executed on 
the result returned by $A d d(10)$. (This interpretation is very much like the dot notation used in $\mathrm{C}++$.) As an example, Axiom (3) states that executing the method Create, followed by Add(v), followed by Tail produces an object that should be (in a correct implementation) equivalent to the list object produced simply by executing Create. Similarly, Axiom (7) says that given a list object L, when executing Add ( $v$ ) on $L$ followed by Length, the result returned by Length should be the same as the result returned by executing Length on $\mathrm{L}$ and adding 1 to the result.

Axioms may be used to derive one sequence from another. For example, the sequence "Create. Add (10).Add(20).Add(30). Delete(20)" may be reduced to Create.Add(10).Add(30) by repeated applications of Axiom (9) using the following sequence of derivations:

Create.Add (10).Add(20).Add(30).Delete(20) $\Rightarrow$ Create.Add(10).Add(20).Delete(20).Add(30)

$\Rightarrow \quad$ Create.Add (10).Add (30).

By understanding derivations such as this one, it is possible to understand the difference between a constructor and transformer method. Constructors represent the "most primitive" sequences of methods that may be used to produce a particular object state. The axioms should be written in such a way that it is possible to rewrite sequences of constructors and transformers into sequences consisting of constructors only. In a correct implementation, it should be possible to produce states equivalent to every possible object state using only constructors.

We now consider the notion of correctness of a class implementation (e.g., in $\mathrm{C}++$ ) with respect to a given LOBAS specification. We say that two method sequences are specification equivalent if the axioms may be used to derive one sequence from the other. For example, the sequences "Create.Add (10) $\cdot \operatorname{Add}(20) \cdot \operatorname{Add}(30) \cdot \operatorname{Delete}(20) "$ and "Create.Add(10).Add(30)" are specification equivalent as a result of the derivation shown above.

Given the above notion of specification equivalence, we now are able to provide an intuitive definition of the correctness of a class implementation. A class implementation is said to be correct with respect to a given algebraic specification iff for every pair $(S 1, S 2)$ of method sequences, $S 1$ is specification equivalent to $S 2$ iff the executions of $S 1$ and $S 2$ within the implementation return "equivalent" values. Two possibilities exist for the types of the values returned by those sequences:

- The values could be from a built-in type (e.g., integer, character, boolean, float), or

- The values could be objects of a user-defined class.

The notion of equivalence between values of built-in types is simple, and may be determined simply by using the built-in equality operator within the implementation language. The notion of equivalence between objects of user-defined classes is much more complicated. In [1], a formal, theoretical definition of object equivalence is given, known as observational equivalence. The basic idea is that two objects are observationally equivalent if those objects cannot be distinguished in client code. One possible heuristic for simulating observational equivalence is to implement an Equal method for the class, whose behavior is similar to that of an equality operator for built-in types. We discuss the use of such a heuristic in the next section. 


\subsection{An Algebraic Specification-Based Test Execution Model}

In this section, we consider a model for test execution based on algebraic specifications. We assume a model similar to that of the ASTOOT system [1]. In this model, a test case is a triple of the form (S1, S2, tag), where $S 1$ and $S 2$ are method sequences and the tag is either "equivalent" or "inequivalent," depending on whether or not the sequences are specification equivalent.

For the moment, we will assume that specific test cases have already been generated. (The problem of generating test cases will be addressed later in the paper.) Given that test cases have already been created, we identify the following basic testing process:

1. For each test case, execute $S 1$ and $S 2$ and determine whether or not they return either "equivalent" values (the mechanism for assessing this equivalence is discussed below).

2. If the test case is to be considered "OK" (unrevealing of a defect), then either (a) the test case tag is "equivalent," and values returned by $S 1$ and $S 2$ are actually "equivalent" or (b) the test case tag is "inequivalent" and the values returned by $S 1$ and $S 2$ are actually "inequivalent." Otherwise, the test case reveals a defect.

We now discuss the mechanism for assessing the equivalence of two sequences when executed as part of a given implementation. Provided that $S 1$ and $S 2$ return values of a built-in type, a test driver can be constructed that automatically compares return values and prints a message as to whether or not the test case reveals a defect. In particular, consider the test case $(B 1, B 2$, equivalent), where $B 1$ and $B 2$ are as follows:

$$
\begin{aligned}
& \text { (B1) Create.Add (10).Add(20). Length } \\
& \text { (B2) Create.Add (10).Add (20).Add(30). Tail.Length }
\end{aligned}
$$

It is easy to verify that $B 1$ and $B 2$ are specification equivalent, which implies that the "equivalent" tag is valid for this (hypothetical) test case. Since Length returns an integer (which is a built-in type), a test for correctness is simply a test to determine whether the integers returned in both sequences are identical. Thus, we have the following test driver psuedocode:

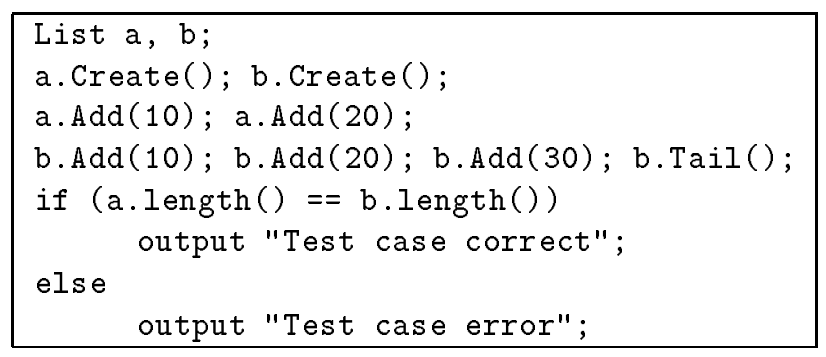

The condition $\mathrm{a}$.length ()$==\mathrm{b}$. length() uses the built-in integer equality operator to determine whether or not the integer results of the two sequences are identical.

On the other hand, if the two sequences both return objects, then a user-defined Equal method must be used to measure equivalence, as was discussed in the previous section. Unlike the built-in 
equality operator (which should not contain defects), this approach is not fool-proof, in that the user-defined Equal method may itself contain errors. However, the testing process will (ideally) reveal any such errors, e.g., if a test case fails and there is no defect in the methods that are explicitly a part of the test, the defect must be in Equal.

The test driver constructed in a situation where the sequences return objects is conceptually the same as the test driver above. In particular, consider the test case (C1, CQ, equivalent), where $C 1$ and $C 2$ are simple modifications of $B 1$ and $B 2$ above (length has been removed):

(C1) Create.Add (10) . Add (20)

(C2) Create.Add(10).Add(20).Add(30). Tail

$C 1$ and $C 2$ are indeed specification equivalent, given that both lists are ordered. Thus, the "equivalent" tag in this test case is valid. Unlike $B 1$ and $B 2$, both $C 1$ and $C 2$ return lists, i.e., objects of a user-defined class. The driver will need to utilize a user-defined list equality method to compare the stack results. In $\mathrm{C}++$, the $==$ operator may be overloaded for the list class. After performing such overloading for class List, the resulting test driver appears below:

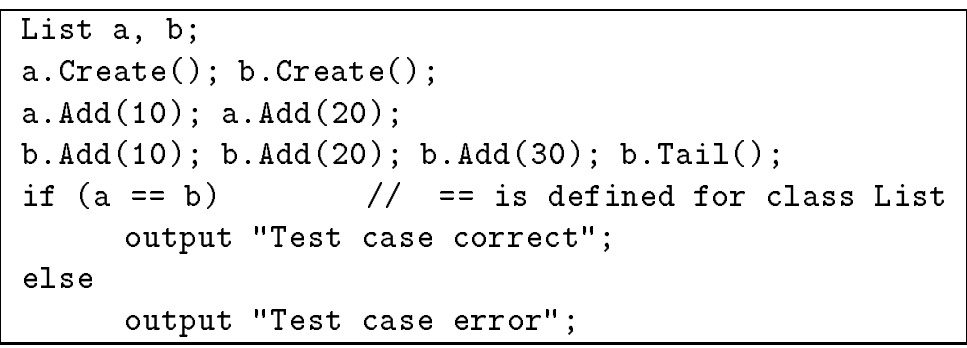

Currently, our method for generating test cases (discussed in Section 3) only generates test cases with equivalent tags. Because of this, we will drop the tag notation in the remainder of the paper, and express test cases as simple pairs of method sequences. As is noted in [1], this eliminates some test cases that may be useful in revealing defects. (The resulting model is actually similar in power to the DAISTS model [2].) However, there are a number of useful test cases still available, and our method is not intended to be the final testing method used when validating a class, but is simply a tool to use during development to assist in defect elimination.

\section{$3 \quad$ Micro-Incremental Development}

Existing class testing techniques assume that the class under test has already been constructed. A completed class normally involves a plurality of methods. In a good design, we would not expect individual methods to be complex [8]. However, many methods are non-trivial, and methods (whether simple or complex) may interact to produce objects whose states are relatively complex. Moreover, under a typical development model, the number of failures that occur initially during testing is relatively high. Tracking down the sources of these failures among a large number of methods that are interacting in potentially subtle ways can prove to be extremely difficult. 
We believe that one of the keys to effective debugging support is in the idea of state simplification. When producing class objects for testing and debugging purposes, objects with simple states are easier to reason about than are objects with complex states. Not all defects are revealed by simple state objects; however, many defects revealed by complex state objects are also revealed by simple state objects. Thus, we are advocating an organization of the class testing process, where testing progresses from (initially) examining simple state objects to (eventually) examining objects with states that are more complex.

We identify two possible "state complexity" dimensions around which to organize an incremental class testing process:

- Structural complexity: The number of different methods that are invoked to produce a given state.

- Behavioral complexity: The number of method invocations producing a given state.

For example, consider the state produced by invoking "Create.Add(10).Add(20).Delete." The structural complexity of this state is 3 , since there are three methods invoked to produce this state (Create, Add, and Delete). The behavioral complexity of this state is 4 (the length of the sequence).

The goal of our incremental testing process can now be more precisely stated: To locate each class defect using the simplest possible state (in terms of both its structural and behavioral complexity). Our process may be characterized as follows:

1. Execute a series of test cases, where the objects produced by the test are of progressively increasing complexity.

2. When a test case in the series fails, cease testing until the defect is repaired.

3. Repairing the defect involves modifying one or more methods. Repeat any previously executed test cases that involve modified methods.

4. Continue testing in this manner until the next defect is revealed (at which point steps 2 and 3 are repeated) or testing is completed without revealing more defects.

This process results in two tangible benefits:

- Reasoning about the source of a defect may be conducted in the simplest possible context.

- Regression testing resulting from defect repair is simplified. In particular, if a defect is located and repaired in the simplest possible state, then the number of methods that have to be reexecuted when retesting simpler states is correspondingly reduced.

We now address the actual mechanisms used to achieve our goal of producing test cases in increasing order of structural and behavioral complexity. First, to ensure a progression of increasing structural complexity during test case generation, we need to be able to test iteratively during the development process. That is, we want to be able to test subsets of methods as those methods are developed. With our (algebraic) specification-based testing model, the order in which methods 
are developed directly impacts the amount of testing that can take place prior to class completion. Thus, a technique is needed to generate the optimal ordering in which methods should be developed for a given class, if we wish to maximize testing during development.

Second, to ensure test case generation is performed in increasing order of behavioral complexity, we need to be able to progressively increase the number of method invocations involved in test cases. Our method for generating test cases directly addresses this.

The remainder of this section is organized as follows. Section 3.1 discusses a method for generating an optimal ordering in which methods should be developed, thus addressing the structural complexity dimension. Section 3.2 discusses a method for generating test cases in increasing order of behavioral complexity, thus addressing the behavioral complexity dimension. Finally, Section 3.3 discusses the integrating these techniques to define an overall testing methodology.

\subsection{Selecting a Development Ordering}

A development ordering is an arrangement of class methods that identifies the order in which the methods should be developed. As discussed above, the selection of such an ordering is determined using the goal of permitting as much testing as possible as early as possible. This allows testing to take place as (small) subsets of methods are written, as opposed to deferring testing until after all methods have been coded.

Not all development orderings are equally good in terms of permitting periodic testing. To illustrate, consider the following specification for a simple stack class:

\begin{tabular}{|l|}
\hline class Stack \\
export \\
Create, Push, Pop, Top \\
constructor \\
Create; \\
Push(v: Integer); \\
transformer \\
Pop; \\
observer \\
Top: Integer; \\
var \\
s: Stack; \\
v: Integer; \\
axiom \\
(1) not (s.Push(v) = Create) \\
(2) s.Push(v).Pop = s \\
(3) s.Push(v).Top = v \\
\hline Table 2: Integer Stack Specification \\
\hline
\end{tabular}

Now consider the development ordering Create $\rightarrow$ Pop $\rightarrow$ Top $\rightarrow$ Push. In this case, no testing is permitted until all methods are complete. In order to construct test cases using a given axiom, all of the methods in that axiom must be present; however, Push is present in every axiom. Thus, it is impossible to construct any executable test cases for the class until after all methods are 
completed (since Push is constructed last). Create $\rightarrow$ Push $\rightarrow$ Pop $\rightarrow$ Top would be a much better development ordering, in that there are opportunities for testing earlier (and more often) in the development process. With this ordering, the developer could:

1. Develop Create and Push and then construct test cases using axiom 1.

2. Test the methods Create and Push.

3. Develop Pop and then construct test cases for Create, Push and Pop using Axioms 1 and 2.

4. Test the method Pop, and perform additional tests on Create and Push. (Additional testing on Create and Push might be necessary because of the new states generated by including Pop in the test cases.)

5. Develop Top and then construct test cases for all four methods using all three axioms.

6. Test all methods in the class.

More generally, given a development ordering $M_{1}, M_{2}, M_{3}, \ldots, M_{n}$, we attempt to maximize:

1. The number of $M_{i}$ s for which new testing is possible (i.e., testing that was not possible to perform after constructing $M_{i-1}$ ), and

2. For each $M_{i}$ after which testing is permitted, the number of axioms available for constructing test cases.

Item (1) deals with maximizing the number of opportunities in which testing may occur, while item (2) deals with maximizing the amount of testing that can take place at a given opportunity. We call an opportunity to test a test point. A test point at position $i$ in a development ordering means that it is possible to construct and execute test cases after developing the $i$ th method that were not executable after the $i$ - 1 th method in the ordering. In the stack example, with the ordering Create $\rightarrow$ Push $\rightarrow$ Pop $\rightarrow$ Top, there are test points after Push, Pop and Top; each method introduces new testing opportunities, as additional axioms may be used in the production of test cases. There is no test point after Create because no test cases can be produced from the axioms with just the Create method.

By developing methods in an order which maximizes both the number of test points and the amount of testing which may occur at a given test point, we are able to design the testing process so that testing can take place on subsets of the methods. This allows us to test in the context of reduced structural complexity. As methods are added, we increase the structural complexity of our test cases in a gradual, orderly fashion.

We now provide a simple greedy-style algorithm to select development orderings in this fashion. To define this algorithm, we first have the notion of an axiom-method table. This table is simply a dynamic table of those methods that appear in each axiom that have not yet been selected for the recommended development ordering. That is, position (1) in the table initially contains those methods that appear in Axiom (1); position (2) initially contains the methods that appear in Axiom (2), etc. 
In order to conduct any testing at all using our model, it is necessary to have two special methods: a method that returns objects "from scratch" and an equality operator. We call methods that return objects without requiring an input object a base constructor. We assume that a base constructor and an equality operator are both developed first, and thus omit them from the axiom-method table. To avoid confusion, we call the base constructor Create and the equality operator Equal, although in our target implementation language, it is possible to implement both as overloaded operators (EQUAL as $==$, and Create as a $\mathrm{C}++$ constructor). We consider this issue further in Section 4.

To illustrate the axiom-method table, we reconsider the ordered list specification from Table 1. In this case, the only base constructor is Create. Although no equality operator is specified, one must still be developed to test this class using our test execution model. However, neither Create nor the equality operator will appear in the axiom-method table for this class. Each position in the table contains the methods that appear in the the axiom corresponding to that position (minus Create). The initial value of the axiom-method table appears below:

\begin{tabular}{|l|l|}
\hline Axiom & Methods \\
\hline$(1)$ & Add, Head \\
$(2)$ & Member \\
$(3)$ & Add, Tail \\
$(4)$ & Add, Head \\
$(5)$ & Add, Head, Tail \\
$(6)$ & Add, Member \\
$(7)$ & Add, Length \\
$(8)$ & Delete \\
$(9)$ & Add, Delete \\
\hline \multicolumn{2}{|l|}{ Table 3(a): Ordered List Axiom-Method Table } \\
\hline
\end{tabular}

The second important concept that we must introduce is the notion of a method table. The method table contains a record for each method with three attributes: (1) the method name; (2) the method's test point contribution and; (3) the method's axiom contribution. A method's test point contribution is simply "yes" or "no," depending on whether a test point is created if that method is chosen. A method's axiom contribution is the sum of the contributions that the method makes to each axiom in which it is used. The notion of "contribution to an axiom" is explained below. The method table is also dynamic, in that once a method is selected for a development ordering, the method table is updated to reflect changes that have occurred with respect to the contribution of the other methods.

The initial value of the method table for the ordered list class is shown in Table $3(\mathrm{~b})$.

The term base constructor is used to distinguish such methods from the LOBAS notion of a constructor, which is slightly different. Base constructors are very much like the $\mathrm{C}++$ notion of a constructor. 


\begin{tabular}{|l|c|c|}
\hline Method & TC & AC \\
\hline Add & no & $3.3 \overline{3}$ \\
Delete & yes & 1.50 \\
Head & no & $1.3 \overline{3}$ \\
Tail & no & $0.8 \overline{3}$ \\
Member & yes & 1.50 \\
Length & no & 0.50 \\
\hline \multicolumn{2}{|l|}{ Table 3(b): Ordered List Method Table } \\
\hline
\end{tabular}

As discussed above, test point contributions (TC) are obtained by noting which methods would result in test points, if chosen as the next method in the development ordering. Member and Delete both result in a test point so the test point contribution for both of these methods is "yes". On the other hand, Add, Head, Tail and Length would not result in a test point if chosen first, so their test point contributions are "no." Axiom contributions (AC) are based on the cumulative contributions of that method to each axiom. For example, Head (which appears in 3 axioms) receives a $1.3 \overline{3}$, based on the sum of:

- 0.5 from Axiom 1, because it one of two methods present (and represents one-half of the methods remaining to be implemented in order to test using Axiom 1).

- 0.5 from Axiom 4, because it one of two methods present (and represents one-half of the methods remaining to be implemented in order to test using Axiom 4).

- $0.3 \overline{3}$ from Axiom 5, because it is one of three methods present (and represents one-third of the methods remaining to be implemented in order to test using Axiom 5)

We then have two selection criteria for the next method:

- First, identify methods that make test point contributions, thus permitting an opportunity to test after that method is constructed. For the above method table, this rule implies we should only consider Delete and Member as the next method.

- Second, for the methods identified in the previous step, choose the next method based on the highest axiom contribution, thus maximizing the amount of testing that can be conducted at a given opportunity. If two methods both define a new test point and both contribute equally, an arbitrary choice can be made. In this example, both Delete and Member have the same axiom contribution (1.5), and so we can make an arbitrary choice between the two.

We choose Delete for the first method (arbitrarily). We then revise the axiom-method table and the method table, as is shown below. The method chosen (in this case Delete) is removed from the axiom-method table. In addition, the method table is revised as follows: (a) Delete is removed from the table and; (b) the axiom and test point contributions are revised accordingly. The new axiom-method and method tables are then shown in Table 4(a). 


\begin{tabular}{|l|l|l|l|l|}
\hline \multicolumn{2}{|c|}{ Axiom-Method Table } & \multicolumn{3}{|c|}{ Method Table } \\
\cline { 1 - 2 } Axiom & Methods & Method & TC & AC \\
\hline$(1)$ & Add, Head & Add & yes & 3.83 \\
$(2)$ & Member & Head & no & $0.8 \overline{3}$ \\
$(3)$ & Add, Tail & Tail & no & $0.8 \overline{3}$ \\
$(4)$ & Add, Head & Member & yes & 1.50 \\
$(5)$ & Add, Head, Tail & Length & no & 0.50 \\
$(6)$ & Add, Member & & & \\
$(7)$ & Add, Length & & & \\
$(8)$ & & & & \\
$(9)$ & Add & & & \\
\hline Table 4(a): Tables after selecting method Delete \\
\hline
\end{tabular}

Of the entries in the revised method table that make a test point contribution, Add has the highest axiom contribution. Consequently, we take Add to be the next method; the revised tables are illustrated in Table $4(\mathrm{~b})$.

\begin{tabular}{|l|l|l|l|l|}
\hline \multicolumn{2}{|c|}{ Axiom-Method Table } & \multicolumn{3}{|c|}{ Method Table } \\
\cline { 1 - 2 } Axiom & Methods & Method & TC & AC \\
\hline$(1)$ & Head & Head & yes & 2.50 \\
$(2)$ & Member & Tail & yes & 1.50 \\
$(3)$ & Tail & Member & yes & 2.00 \\
$(4)$ & Head & Length & yes & 1.00 \\
$(5)$ & Head, Tail & & & \\
$(6)$ & Member & & & \\
$(7)$ & Length & & & \\
$(8)$ & & & & \\
$(9)$ & & & & \\
\hline \multicolumn{2}{|l|}{ Table 4(b): Tables after selecting method Add } \\
\hline
\end{tabular}

At this point, Head has the highest axiom contribution of those methods making a test point contribution, so it is chosen next. Repeatedly applying this same procedure to the remaining methods, the final ordering (including Create and Equal) is Create $\rightarrow$ Equal $\rightarrow$ Delete $\rightarrow$ Add $\rightarrow$ Head $\rightarrow$ Tail $\rightarrow$ Member $\rightarrow$ Length.

This process focuses primarily on subsets of the methods in which it is still possible to conduct testing, thus allowing us to initially test in the context of structurally simple states involving a limited number of methods. This testing using simple states may then gradually transition to testing that involves more complex states.

\subsection{Generating Test Cases}

Our method for generating test cases involves generating "object states," and then using those states as inputs to axioms from the specification. Thus, we divide the test case generation problem into two parts: the problem of generating object states and then the problem of actual test case construction, using the object states as inputs.

In [10], we first described a relatively simple scheme for generating object states. The basic idea is to identify those methods that have an effect on the object state. In LOBAS terms, these are 
the constructor and transformer methods. In the case of the ordered list class, those methods are Create and Add (constructors) and Delete, Head and Tail (transformers). It is useful to further subdivide this group of methods in a slightly different way. Recall from Section 3.1 that a method is a base constructor if it produces an object from scratch, i.e., without taking an object as input. To develop our state generation approach, we will need to segregate base constructors from other constructor/transformer methods. We call the other constructor/transformer methods modifiers, in that they take an object as input and modify the object to produce a result.

It is then possible to systematically generate a series of increasingly complex object states. These states may be used as input to the axioms during testing, as is described later in this section. To organize the state generation process, we utilize what we call a state tree. Each node in the tree represents a particular state. The character strings at each node represent the method sequences used to generate the state (e.g., CAD refers to the state generated by executing Create, followed by Add, followed by Delete). A partial state tree for our list class is shown in Figure 1.

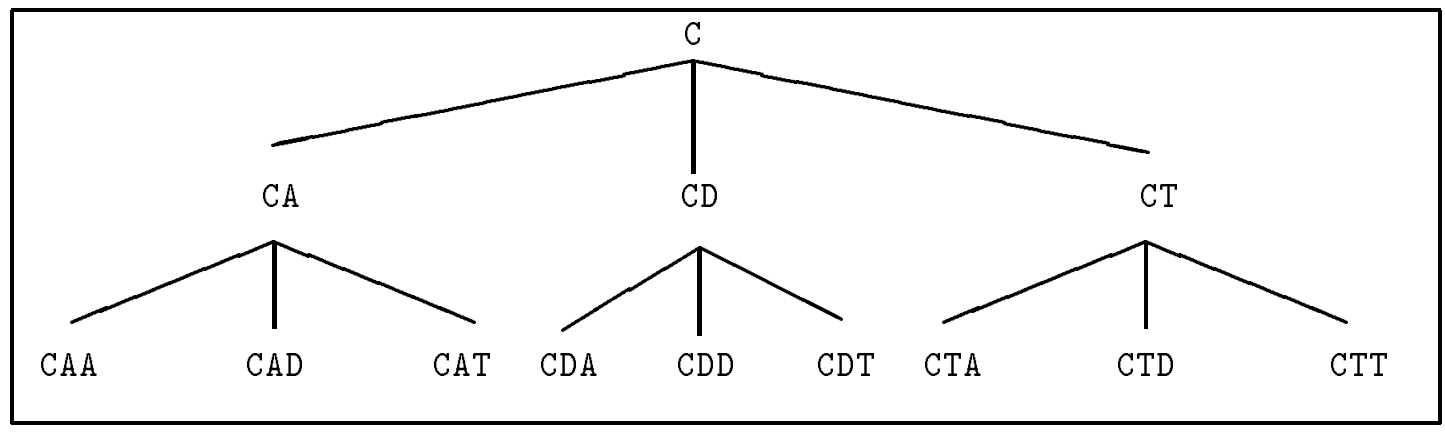

Figure 1: State Tree for Ordered List Class

The states in the tree are generated as follows. First, the base constructor method is executed to generate the initial (root) state. Next, the states at Level 1 are generated, from left to right, by invoking the methods at each node. Following the generation of Level 1 states, the states at each successive level are generated in similar fashion.

It should be noted that other information is needed to generate states than simply the order of method invocations as defined within the state tree. In particular, the sequence CAD indicates that Create, Add and Delete are to be executed on some defined list object. In $\mathrm{C}++$ syntax, the object is written in front of the method in an invocation (e.g., the L in L.Add(v)). However, each of these methods (Create, Add, Delete, and Tail) may take other "secondary" parameters. For example, Add and Delete take a specific element value that is to be added or deleted (e.g., the $\mathrm{v}$ in L.Add (v)). In general, there are three types of secondary parameters that can be expected in a given implementation:

- Parameters of built-in types, such as integer, character, real, boolean, etc.,

- Parameters of a class other than the class under test, and 
- Parameters of the class under test. In the case of a "binary" method (e.g., concatenate, which takes two string objects), one of the two parameters may be viewed as the primary parameter and the other one as the secondary parameter (e.g., s1.concat(s2), where s2 is the secondary parameter). Note that in $\mathrm{C}++$, operator overloading may eliminate the syntactic distinction between primary and secondary parameters (e.g., s1 + s2).

Our current implementation builds a state tree for the primary object parameter, and randomly produces values for secondary parameters. For built-in types, this essentially involves using a random number generator; for class objects, this involves generating random constructor and transformer method sequences. Although this introduces an element of randomness to an otherwise systematic process, we can at least ensure that at least one parameter of the class under test is being systematically manipulated by method invocation sequences of steadily increasing complexity.

States produced by this process are used as parameters to the axioms to generate test cases. Specifically, if there is a variable in an axiom of the type of the class under test, then states from the state tree are used to produce values for the variable in constructing test cases. For example, consider Axiom (7) from the ordered list specification in Table 1:

\section{L.Add(v). Length $=$ L. Length +1}

Producing test cases from this axiom involves generating a succession of values for $\mathrm{L}$ and $\mathrm{v}$. Using our state tree generation strategy, we can have the following sequence of values that may be substituted for $\mathrm{L}$ (the first three levels of the state tree):

\begin{tabular}{|ll|}
\hline L1 & Create \\
L2 & Create.Add(10) \\
L3 & Create.Delete(6540) \\
L4 & Create.Tail \\
L5 & Create.Add(10).Add(564) \\
L6 & Create.Add(10).Delete(430) \\
L7 & Create.Add(10).Tail \\
L8 & Create.Delete(6540).Add(543) \\
L9 & Create.Delete(6540).Delete(54987) \\
L10 & Create.Delete(6540).Tail \\
L11 & Create.Tail.Add(237) \\
L12 & Create.Tail.Delete(10) \\
L13 & Create.Tail.Tail \\
\hline
\end{tabular}

Note that once a value is randomly generated for a parameter, that value is retained for the parameter within a given subtree. For example, the first invocation of Add in every node of the subtree rooted at CA receives the parameter value "10" (i.e., in L2, L5, L6 and L7 in the table above).

The object values from the above table may then be substituted back into the axiom to create a series of test cases: 


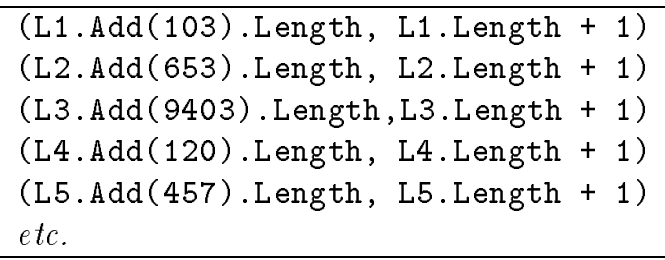

The progression of states from the state tree is substituted for the leftmost list variable to appear in each axiom. Substitutions for all other variables are made at random. However, by using the progression of states generated by our state tree to make at least one substitution, we normally meet our goal of ensuring that test cases steadily increase in complexity. We acknowledge, however, that due to the randomness of the remaining substitutions, there will be some exceptions.

The same substitution process takes place for the remaining axioms to generate additional test cases. We note that, for branching axioms (i.e., axioms that contain an if or some type of conjunction with multiple exclusive parts), there is no guarantee of testing all branches of the axiom; testing both branches will normally require that the random values generated for various variables be appropriate to ensure such coverage. For example, consider the axiom:

L.Add (v).Head $=$ if $v>$ L.Head then $v$ else L.Head

Testing both branches for the right hand side requires that values be generated for $\mathrm{v}$ and $\mathrm{L}$ such that $\mathrm{v}>\mathrm{L}$. Head and $\mathrm{v}<=\mathrm{L}$. Head. It is possible that (eventually) such values will be generated simply by generating a large number of test cases. However, since this cannot be guaranteed, our environment (as discussed in Section 4) produces information regarding which branches of the branching axioms have been tested. In this way, the developer can monitor whether additional testing is needed.

A final question which surfaces involves determining when to stop testing. As we discuss in Section 4.1, the developer must specify two things when using our environment to generate test cases: (a) the number of levels in the state tree and (b) which base constructors to use in generating state trees (as it is possible to generate a distinct state tree for every base constructor). However, the testing technique does not provide any guidance regarding how to make these two choices; effectively, there is no stopping criterion built into the testing technique. We would expect that a necessary condition for stopping would be coverage of all branching axioms, as discussed above. This is consistent with the approach used by DAISTS [2]. However, we certainly do not feel that this is a sufficient condition.

On the other hand, we are not suggesting that this method should be the only testing method applied to a class, where a decision would have to be made based solely on our results regarding whether or not the class had been tested adequately. Instead, this method is a development and debugging-oriented approach that makes an initial pass at testing classes in an organized fashion during development. Once the class is completely developed, a more rigorous testing method with a definite completion point could still be applied to the completed class (such as the technique from 
[1]). Further investigation is needed to determine how extensively testing should be conducted during development, as well as to determine ways of refining or modifying our test generation strategy. One of the advantages of our overall framework is that it is possible to modify the test generation strategy (or the development ordering generation strategy) and still retain the overall benefit of incremental development within a general framework.

\subsection{An Overall Development and Testing Methodology}

Our method for generating test cases (in Section 3.2) may be combined with our method for generating development orderings (in Section 3.1) to create an overall testing approach in the context of development. In particular, suppose that you have the following development ordering for the ordered list class as defined in Section 3.1 (Equal $\rightarrow$ Create $\rightarrow$ Delete $\rightarrow$ Add $\rightarrow$ Head $\rightarrow$ Tail $\rightarrow$ Member $\rightarrow$ Length). A general development and testing process would be as follows:

1. Develop Equal, Create and Delete and generate test cases using Axiom 8 and state tree generation restricted to these two methods. In particular, the state tree would be linear, with Delete the only modifier method in the tree (the generated states would be C, CD, CDD, CDDD, CDDDD, etc.).

2. Develop Add and generate test cases using Axioms 8 and 9. In this case, the state tree would be binary, with Add and Delete as modifiers and Create as the base constructor. States are produced such as $\mathrm{C}, \mathrm{CA}, \mathrm{CD}, \mathrm{CAA}, \mathrm{CAD}, \mathrm{CDA}$, and $\mathrm{CDD}$ (the first three levels in the state tree in breadth-first order).

3. Develop Head and generate test cases using Axioms 1, 4, 8 and 9. At this point, we have three modifier methods (Add, Delete and Head), and so the state tree is ternary. States produced include C, CA, CD, CH, CAA, CAD, CAH, CDA, CDD, CDH, CHA, CHD, CHH (again, the first three levels in breadth-first order).

4. Develop Tail and generate test cases using Axioms 1, 3, 4, 5, 8 and 9. Now we have four modifier methods (Add, Delete, Head, and Tail), and the state tree is extended appropriately.

5. Develop Member and generate test cases using Axioms 1, 2, 3, 4, 5, 6, 8 and 9. Although we are able to conduct testing using additional axioms beyond the previous step, the state tree does not change from before, since Member is not a modifier method.

6. Develop Length and generate test cases using all of the axioms. Again, the state tree remains as before; however, we are able to generate additional test cases via the use of Axiom 7 .

Thus, we achieve our first goal of incremental structural complexity by "testing as we go," before all of the methods are written, limiting the number of methods that can appear in test cases to just those which have been written. We achieve our second goal of incremental behavioral complexity by utilizing a progression of states from a state tree as object inputs to the axioms in constructing test cases. 


\section{An Integrated Development Environment}

\subsection{Environment Functionality and Architecture}

An integrated environment which supports the class development paradigm described in Section 3 is presently under construction; we currently have a working prototype that we are using for experimental purposes. In our environment, LOBAS specifications are used as input to generate three separate items:

- A development ordering for the class,

- A "template" for a (partial) class (using $\mathrm{C}++$ ), and

- A test driver program for a (partial) class.

The notion of a development ordering was discussed extensively in Section 3. A test driver program is just a program that executes the test cases generated for the class using the techniques of Section 3.2. The notion of a class template is discussed below.

Let us first consider the sketch of the environment's X-Windows interface found in Figure 2. Figure 2 assumes that we have loaded the stack specification from Table 2 (Section 3.1). We can generate the development ordering by clicking on the Ordering button, thus resulting in the configuration shown in Figure 3.

Now we are in a position to begin development. We begin by clicking the buttons containing the names of the methods that we wish to develop from the top of the screen. Clicking on Create and Push indicates that we wish to develop these two methods first (as the ordering suggests). Once we have clicked on both methods, Axiom (1) shifts from the "Inactive" to "Active" windows. Figure 4 shows the configuration of the development manager at this point. This configuration indicates visually that we have a "partial specification" consisting of Axiom (1) now available for test generation purposes. Thus, once Create and Push are actually written, we can conduct testing using Axiom (1) to generate test cases.

To assist in writing Create and Push, we first click on the C++ code button. This generates a "template" for the C ++ class, based on information found in the LOBAS specification, as follows:

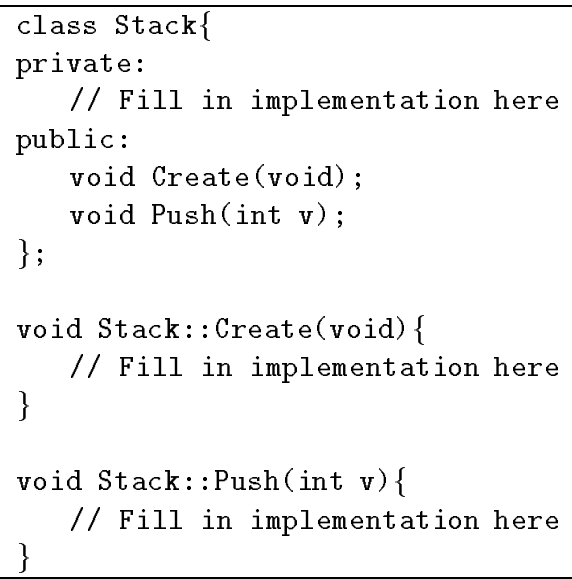




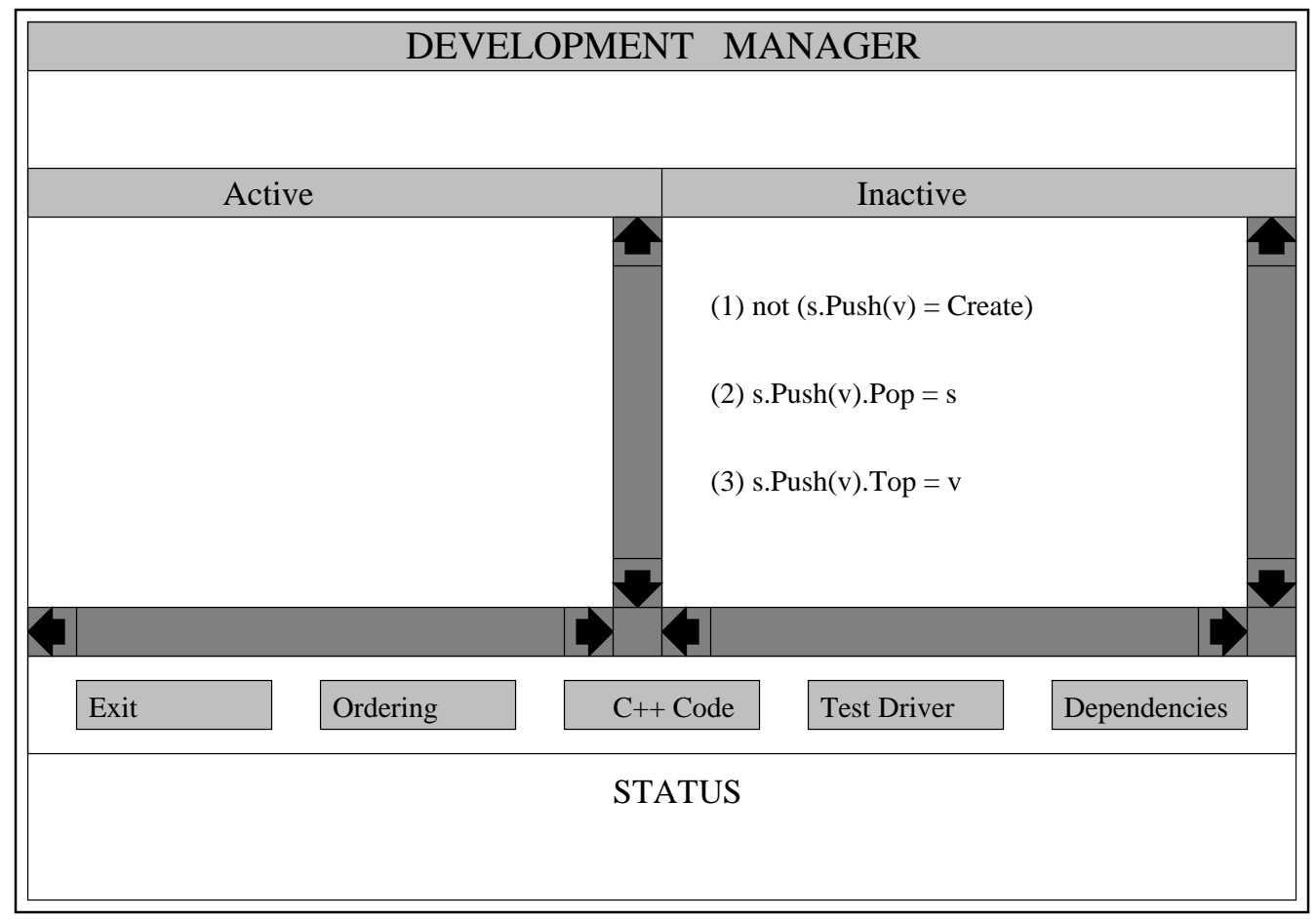

Figure 2: Initial Configuration

Our current implementation simply takes the method names from the LOBAS specification and generates the $\mathrm{C}++$ template accordingly. However, it should also be possible to specify mappings from LOBAS names to $\mathrm{C}++$ names. For example, it would be appropriate to implement Create as a $\mathrm{C}++$ constructor rather than as a method called Create. We are currently working on an enhancement to the prototype that provides the facility to specify such mappings.

At this point, the developer must provide an implementation for the actual data representation for class Stack, and then implement Create and Push. Once bodies are written for these methods, testing may begin. Clicking on Test Driver generates another window, where the user is asked to specify two things:

- The base constructors for which it is desired to generate state trees (effectively, how many different state trees should be generated) and,

- The number of levels in each state generation tree that should be generated.

Once this information is provided, a test driver is generated. This driver executes the test cases produced by combining the state trees with the axioms, as described in Section 3.2. For every test case, the driver executes the two sequences found in the test case, and then compares the results of those sequences to determine whether or not the test case passes (as discussed in earlier sections). Information regarding any test cases that fail is shown in a separate window. Also, whenever a test 


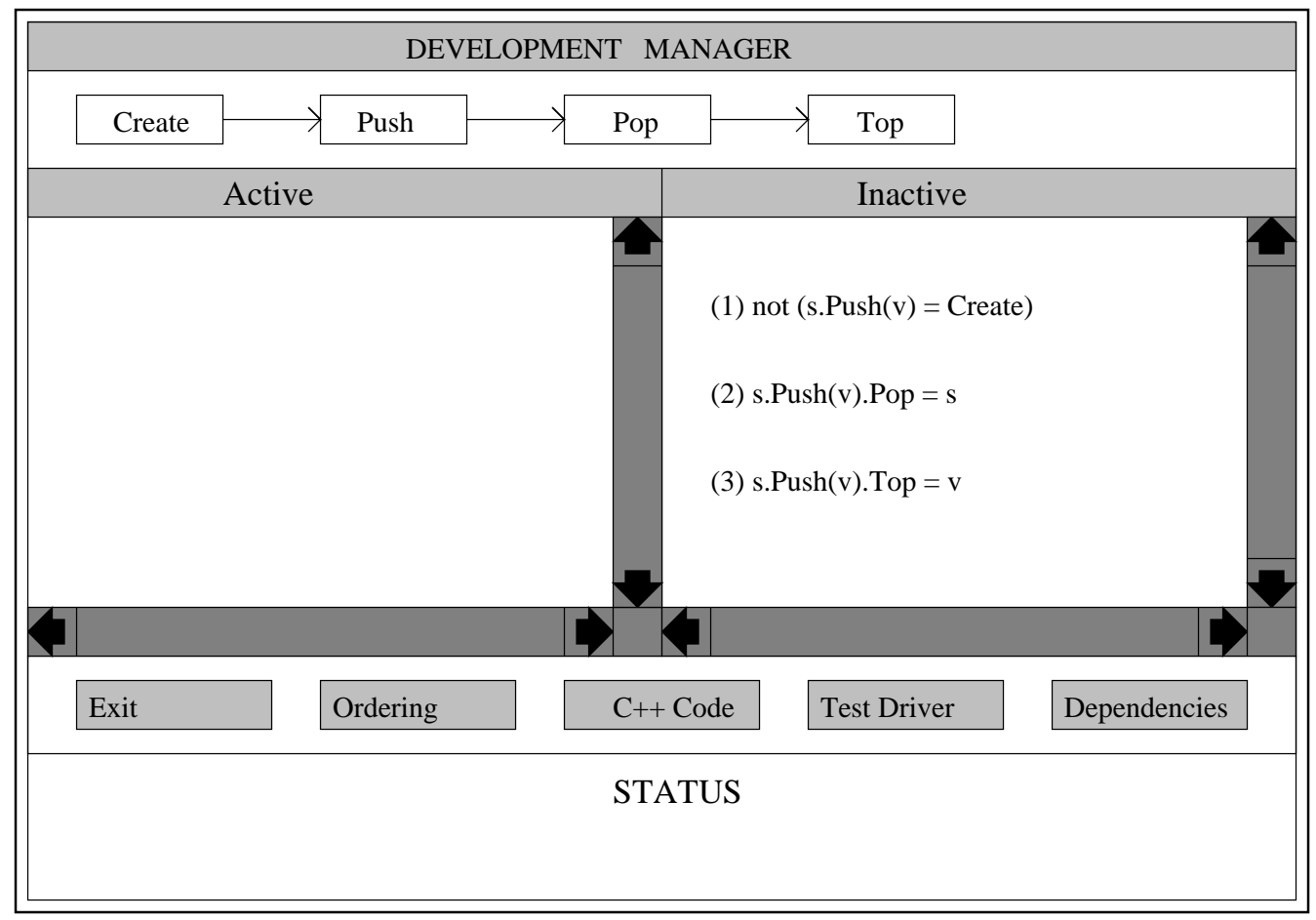

Figure 3: After Generating Development Ordering

case is executed that involves a branching axiom, information regarding which branch of the axiom is actually tested is also output to the separate window.

Once Create and Push have been debugged, the tester can click on additional methods. Clicking on Pop causes Axiom (2) to become active, meaning that more testing can now be performed. Clicking on C++ Code extends the existing class with (a) a prototype for the Pop method and (b) a function definition for Pop with an empty body. The developer may fill in the contents of Pop, click on Test Driver, and continue testing as before.

The only remaining button not discussed so far is the Dependencies button. Clicking on Dependencies allows the developer to define implementation dependencies among the methods. For example, suppose the developer wishes to implement Top by invoking Pop on a local copy of the stack and returning the value that is removed. Consequently, there is a dependency in the implementation (not present within the specification) that needs to be specified. Figure 5 contains the window where implementation dependencies are defined.

By specifying this dependency, the development ordering generator will not allow an ordering to be constructed that contains Top before Pop. The actual implementation simply will not set Top's test point contribution to "yes" until Pop has been selected. It should be pointed out that such an implementation may cause the development ordering algorithm to fail, because there may be a case where the only method making a test point contribution depends on some method that hasn't been developed yet. In such a case, the developer is told of the conflict and the reasons 


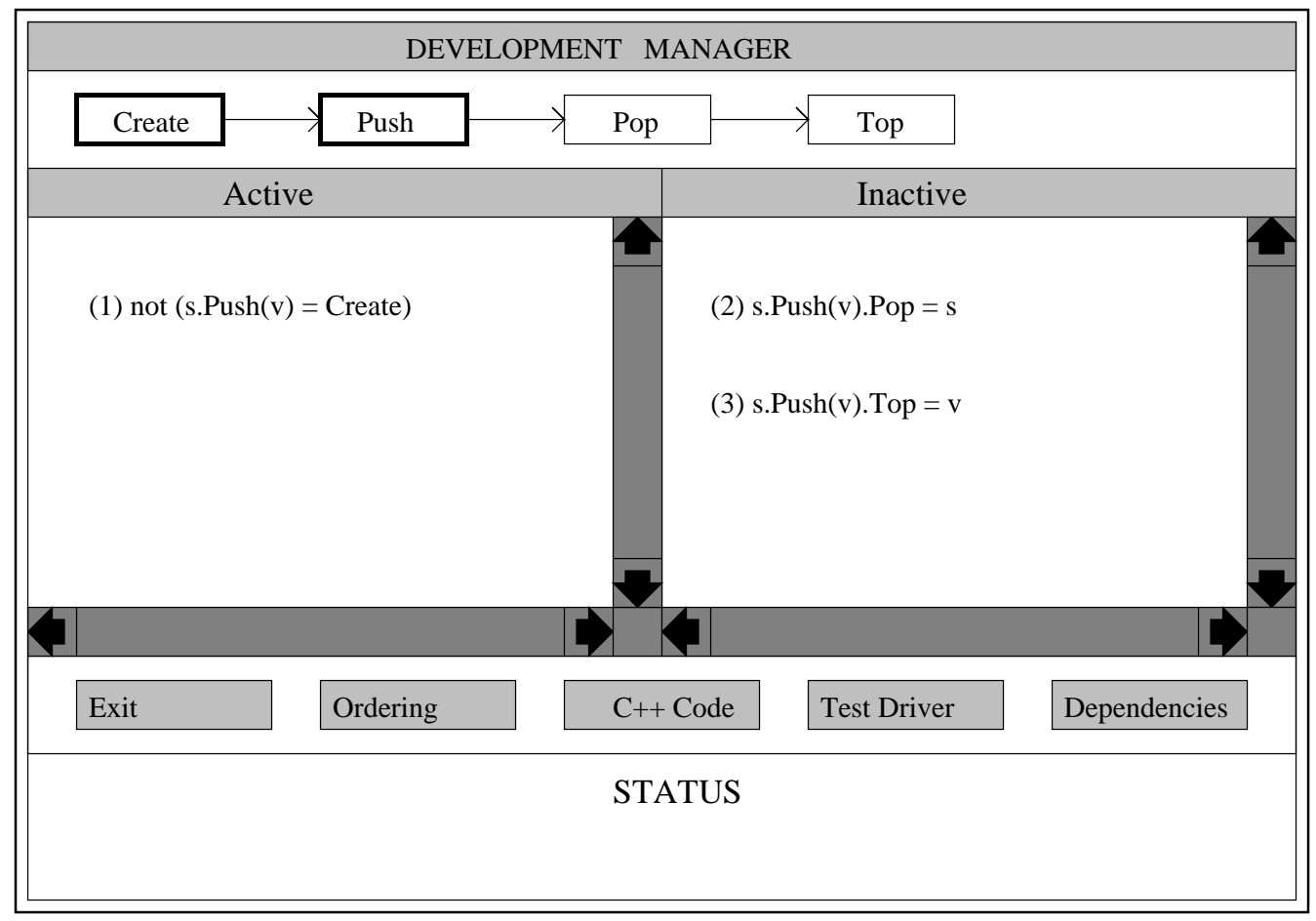

Figure 4: After Selecting Create and Push

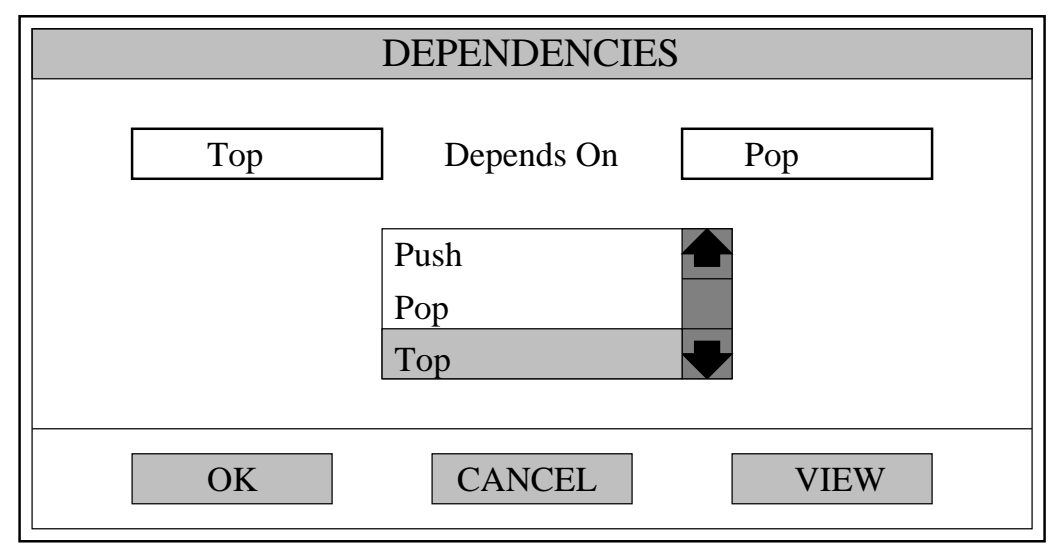

Figure 5: Defining Implementation Dependencies 
for it, and is given the opportunity to resolve the conflict by either changing the specification or removing the implementation dependency. Failure to resolve the conflict simply means that testing will have to be deferred until the class is completed.

\subsection{Case Study: Building A List Implementation}

As an example illustrating the utility of this environment, we developed a $\mathrm{C}++$ implementation of the ordered list class. For demonstration purposes, we elected to generate test cases utilizing an eight-level state tree. As already discussed, the development ordering generated by the environment (based on our algorithm from Section 3.1) was Create $\rightarrow$ Equal $\rightarrow$ Delete $\rightarrow$ Add $\rightarrow$ Head $\rightarrow$ Tail $\rightarrow$ Member $\rightarrow$ Length.

Our development was completed by one of the authors in a relatively non-contrived setting. In particular, the author was handed the specification and was given the development environment, with no preparation regarding expected outcomes. The mistakes made were not deliberately contrived, but were in fact real. A log of the development process appears as follows:

1. The initial step was to build the first methods in the ordering: Create, Equal (implemented as $=\mathbf{=}$ ) and Delete. No testing was possible until after Delete was completed, as Delete constituted the first test point in the ordering. At this point, we were able to generate test cases using Axiom 8 and an eight-level state tree (i.e., C, CD, CDD, CDDD, etc., up to eight levels). All test cases passed.

2. The method Add was developed, allowing test cases to be generated using Axioms 8 and 9 and an eight-level state tree produced by Create (base constructor) and Delete and Add (modifiers). All test cases passed.

3. The method Head was developed, allowing us to generate test cases with Axioms 1, 4, 8 and 9 (and an eight-level state tree). Executing the test driver resulted in a segmentation fault, which upon subsequent examination, was found to be caused by an uninitialized pointer. The defect was repaired.

4. The test cases from step (3) were repeated. The result was that one of the Axiom 4-based test cases, (Create.Add (27). Add (540). Head, 540), failed. Because of our incremental approach, it can be inferred that the defect is in one of three methods: Create, Add or Head (the only methods involved in the construction and execution of the test case). The cause turned out to be the Add method, in that it did not insert elements in the proper order, but rather simply maintained the list in random order. This defect was repaired.

5. The test cases from step (3) were again repeated. Again, a similar test case (based on Axiom 4) to the one from step (4) failed, although with different random number integer inputs. Similar inferences can therefore be made regarding the source of the defect. The cause was

Note that for some test cases, the class equality operator is also involved in executing the test case; however, the built-in integer equality operator was used in this test case because the two sides of the test case had integer results. 
that Add now inserted the elements into the list in the wrong order (ascending, rather than descending).

6. The test cases from step (3) were again repeated, again with a failure on a test case like the one in step (4) above. The problem this time was that the special case of inserting an element at the head of the list was not handled properly. The defect was repaired and the tests repeated with no failures.

7. At this point, the Tail method was added, allowing test cases to be generated with Axioms 3 and 5 (in addition to Axioms 1, 4, 8 and 9, which were already available). Running the test cases revealed a failure in an Axiom 5-based test case where the integer parameter to Add equaled the item already at the head of the list. (This test case is too complex to specify here, given that generating a parameter to Add that was already at the head of the list was a random event and did not occur early in the sequence of test cases.) This test case was created and executed using Create, Add, Head, Tail and Equal. Thus, Delete was not examined for the defect, nor were Member and Length (which are not even written yet). It was eventually concluded that the defect was the result of an incorrect assumption that if an existing element was to be added again to the list it should not be duplicated. The axioms, however, imply that elements may be duplicated. Thus, Add was modified accordingly.

8. Although Delete was not examined directly in the previous step, we realized at this point that a change to Delete was necessary. The original implementation of Delete removed all elements in the list of a given value, while the revised implementation only removes one such element if there are multiple copies. After making these changes and re-running the test cases from the previous step, no failures were observed.

9. The method Member was added next, allowing test cases to be produced using all of the axioms except Axiom 7. Test cases were then produced using an eight-level state tree and axioms 1-6 and 8-9. No failures were observed when executing any of the test cases.

10. Finally, the method Length was added, allowing test cases using all of the axioms to be generated. Testing was performed using all axioms and all states in an eight-level state tree. No failures were observed.

The important observation to make about this process is that, cumulatively speaking, there were actually six separate defects ultimately observed in this class. Although two of the defects could not have arisen simultaneously (the Add defects where the list was first unordered and then ordered in the wrong direction), five of the defects could have co-existed within an initial version of the class. Isolating and eliminating defects (even relatively straightforward defects such as these) would have been extremely difficult in such a context. Our incremental approach provides the advantage of "divide-and-conquer" when locating and removing defects, which is not available if testing is deferred until the class is complete. 
To appreciate the difference between incremental and "post-development" class testing, consider the fact that there were test cases involving two different axioms that failed in the above testing scenario (Axioms 4 and 5). This allowed us to restrict our attention to analyzing only those axioms, and only the methods involved in producing test cases with those axioms. With post-development testing, the various defects in the Add method would have caused failures to occur in most of the axioms where Add is present (possibly Axioms 4, 5, 6, 7, and 9). In particular, the failure to add an element properly to the head of the list would have resulted in definite failures in test cases based on Axioms 6 and 7 (the axioms which specify Member and Length). Thus, the developer might have wasted time looking for defects in Member and Length when none existed. Instead, we eliminated the Add defect before test cases based on Axioms 6 and 7 were even constructed and executed.

\section{Conclusion}

In this paper, we have identified an incremental development process for object-oriented class development. We call this type of process "micro-incremental" to reflect the idea that it is incremental with respect to developing the internals of a single class. We believe that this type of process is both natural and important to the development of classes, which may contain methods that interact in complex ways. Identifying an incremental process of this type simplifies regression testing and makes the defect removal process more efficient.

Future work will involve the development of additional development ordering and test case generation strategies as alternatives to our current strategies. We acknowledge that there are limitations to the current strategies, and intend to consider alternatives that fit within this paradigm. Our development environment is constructed to provide an overall framework where other testing strategies may be substituted without sacrificing any of the advantages of incremental development. A primary goal of our future work is to experimentally evaluate a number of different incremental development strategies over a wide variety of classes; our environment provides a framework for such evaluation.

\section{References}

[1] Doong, R. and P. Frankl. "Case Studies on Testing Object-Oriented Programs," Proceedings of the Fourth Symposium on Software Testing, Analysis and Verification, October 1991, pp. 165-177.

[2] Gannon, J., P. McMullin, R. Hamlet. "Data Abstraction, Implementation, Specification and Testing," ACM Transactions on Programming Languages and Systems, vol. 3, July 1981, pp. 211-223.

[3] Guttag, J., J. Horning, and J. Wing, "The Larch Family of Specification Languages," IEEE Software, vol. 4, no. 5, September, 1985, pp. 24-36.

[4] Harrold, M., J. McGregor and K. Fitzpatrick. "Incremental Testing of Object-Oriented Class Structures," in Proceedings of the International Conference on Software Engineering, 1992. 
[5] Hoffman, D. and C. Brealey. "Module Test Case Generation," Proceedings of the ACM SIGSOFT '89 Third Symposium on Software Testing, Analysis and Verification, December 1989, pp. 97-102.

[6] Jalote P. "Testing the Completeness of Specifications," IEEE Transactions on Software Engineering, vol. 15, May 1989, pp. 526-531.

[7] Liskov, B. and Guttag, J. Abstraction and Specification in Program Development, McGrawHill, New York, 1986.

[8] McGregor, J. and D. Sykes, Object-Oriented Software Development: Engineering Software for Reuse, Van Nostrand Reinhold, 1992.

[9] Parrish, A., D. Cordes, and H. Dyal. "Incremental Testing of Algebraically Specified ObjectOriented Software Modules," Department of Computer Science Technical Report, The University of Alabama, 1995.

[10] Parrish, A., D. Cordes, and M. Govindarajan, "Systematic Defect Removal from ObjectOriented Software Modules," in Proceedings of the Seventh International Software Quality Week Conference, San Francisco, May 1994.

[11] Zweben, S., W. Heym, and J. Kimmich, "Systematic Testing of Data Abstractions Based on Software Specifications," Journal of Software Testing, Verification and Reliability, Vol. 1 No. 4, Jan-Mar 1992, pp. 39-55. 